\title{
O diaǵnóstico do carcinoma metastático de coróide pela biópsia aspirativa com agulha fina (BAAF) - Relato de caso
}

\author{
The diagnosis of metastaticcarcinoma of the choroid using \\ fine-needle aspiration biopsy(FNAB)-Case report
}

\author{
Zélia Maria da Silva Corrêa ${ }^{1}$ \\ Luciane Cristina Dreher Irion² \\ Ítalo Mundialino Marcon ${ }^{3}$ \\ Raquel Goldhardt $\mathbf{t}^{4}$ \\ Giovanni Marcos Travi ${ }^{5}$
}

\begin{tabular}{|l|}
\hline \multicolumn{1}{|c|}{ RESUMO } \\
\hline Objetivo: Relatar o caso de um paciente com carcinoma de pulmão cuja \\
primeira metástase detectada foi em coróide e a abordagem diagnóstica \\
deste caso. Métodos: Um homem de 35 anos, em tratamento devido a uma \\
condensação pulmonar isolada, referiu baixa da acuidade visual súbita, \\
dor, secreção e olho vermelho (olho direito) há 10 dias. Ao exame oftalmo- \\
lógico foi detectada a presença de descolamento não regmatogênico de \\
retina emúltiplos focos tumorais em coróideconfirmadopelaultra-sonografia \\
ocular diagnóstica. Foi sugerida biópsia aspirativa comagulha fina(BAAF) \\
para diagnosticar possível doença metastática. A biópsia aspirativa com \\
agulha fina foi realizada sob anestesia peribulbar e sedação. A rota \\
escolhida foi transvítrea através de esclerotomia a 4 mm do limbo. O \\
procedimento foi monitorado via oftalmoscopia binocular indireta. Foram \\
obtidas 2 amostras de focos tumorais diferentes. Após a biópsia aspirativa \\
com agulha fina, as amostras foram encaminhadas para processamento, \\
fixação e coloração pelos métodos Papanicolaou e hematoxilina-eosina. \\
Resultados: A citologia confirmou a suspeita de múltiplos focos metastá- \\
ticos coroídeos. Os aspirados ocular e pulmonar revelaram ser de mesma \\
origem devido à reação positiva com pan-citoqueratina (AE1/AE3). O \\
paciente evoluiu para óbito 4 meses após diagnóstico citológico de \\
carcinoma metastático ocular. Conclusões: A biópsia aspirativa com \\
agulha fina foi eficiente em diagnosticar e correlacionar a citologia ocular \\
com o tumor primário por métodos citoquímicos neste caso. A biópsia \\
aspirativa com agulha fina ainda deve ser usada em casos selecionados e \\
pesquisas futuras serão necessárias para que este procedimento diagnósti- \\
co seja considerado padrão em oftalmologia.
\end{tabular}

Descritores: Neoplasias pulmonares/complicaç̃es; Neoplasias da coróide/secundário; Neoplasias da coróide/patologia; Imunohistoquímica; Ultra-sonografia; Biopsia por aguIha/métodos; Olho/citologia; Carcinoma/patologia; Neoplasias oculares/patologia; Hematoxilina/uso diagnóstico; Amarelo de eosina-(YS)/uso diagnóstico; Relato de caso

\section{INTRODUÇ̃̃̃O}

A biópsia aspirativa com agulha fina (BAAF) em lesões intra-oculares tem se mostrado um método confiável e seguro para estabelecer o diagnóstico em casos selecionados ${ }^{(1-7)}$ e tem sido amplamente divulgada para casos duvidosos de tumores intra-oculares ${ }^{(2-3,7)}$, na necessidade de confirmação diagnóstica $^{(3-4)}$ ou para guiar o tratamento adequado ${ }^{(8)}$.

O uso da biópsia aspirativa com agulha fina (BAAF) em oftalmologia foi relatado em alguns artigos nos anos 40 e $50^{(7)}$, mas seu uso mais intenso se 
deu a partir de 1979 com a publicação do artigo de Jakobiek e colaboradores $^{(4)}$. Após uma série de publicações de Augsburger e colaboradores ${ }^{(1-3,5)}$, a BAAF começou a ser amplamente discutida e usada em vários centros de especializados em oncologia oftalmológica.

A indicação precisa da BAAF é muito importante quando se considera este método diagnóstico, uma vez que a sua utilização ainda não está indicada como rotina em todos os casos de tumores intra-oculares ${ }^{(1)}$. A BAAF tem sido realizada em nosso serviço com a aprovação do Comitê de Ética e Pesquisa nos seguintes casos: 1 - incerteza diagnóstica mesmo após todos os exames não-invasivos que se dispõe, 2recusa do paciente em aceitar tratamento mediante o diagnóstico clínico de neoplasia maligna intra-ocular e 3- na suspeita de tumor metastático quando se desconhece a neoplasia primária ou o paciente não tem diagnóstico de doença metastática como no caso descrito a seguir.

\section{RELATO DO CASO}

Um homem branco com 35 anos, inicialmente avaliado no Departamento de Pneumologia devido à dor em parede lateral do hemi-tórax esquerdo e tosse, foi encaminhado ao Serviço de Oftalmologia com queixa de diminuição súbita da acuidade visual em olho direito acompanhado de dor, hiperemia conjuntival e secreção muco-purulenta há 10 dias. Antes da consulta, usou Terramicina pomada (1 vez ao dia) durante dois dias e gentamicina colírio, sem obter melhora.

Sua história pregressa incluía uma radiografia de tórax realizada há 3 meses que mostrava consolidação em lobo inferior esquerdo. Devido a suspeita de pneumonia, o paciente foi tratado com antibióticos, entretanto sem melhora do quadro. Não foram relatados episódios de febre desde o início do quadro pulmonar apesar dos episódios de sudorese noturna. O paciente negou emagrecimento, dispnéia e tabagismo.

Ao exame físico, o paciente apresentou-se em bom estado geral, lúcido, orientado, eupneico, acianótico, hidratado, anictérico. Sinais vitais estavam estáveis. A ausculta pulmonar revelou diminuição do murmúrio vesicular na metade inferior esquerda. Não foram detectadas quaisquer alterações em outros sistemas.

No exame oftalmológico inicial (03/08/1999), a acuidade visual medida era: $\mathrm{OD}=\mathrm{MM} 30 \mathrm{~cm}$ e $\mathrm{OE}=20 / 20$. Na biomicroscopia do segmento anterior observou-se, no olho direito, uma hiperemia conjuntival 3 a 4+, secreção mucosa abundante e pupilas pouco reagentes à luz. $\mathrm{O}$ olho esquerdo apresentouse dentro dos limites da normalidade. (Fig. 1) A pressão intraocular (PO) medida foi: $\mathrm{OD}=19 \mathrm{mmHg}$ e $\mathrm{OE}=11 \mathrm{mmHg}(13: 40 \mathrm{~h})$. A oftalmoscopia binocular indireta revelou descolamento de retina não-regmatogênico e coróide elevada irregularmente havendo a suspeita de massa intra-ocular em OD (Fig. 2A). Devido ao extenso descolamento exsudativo da retina não foi possível avaliar com segurança outras características da lesão como coloração, presença de pigmentos em superfície e for-

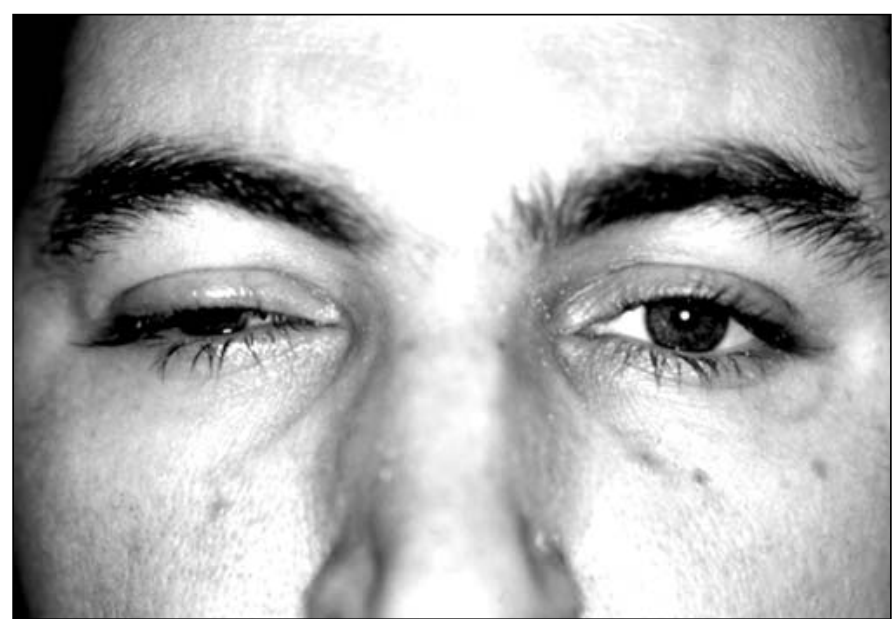

Figura1 - Fotografia com o aspecto dos olhos na primeira consulta. Note o edema e hiperemia palpebral direita. Na biomicroscopia do segmento anterior observou-se hiperemia conjuntival 3 a 4+, secreção mucosa abundante e pupilas pouco reagentes à luz

mato da lesão. OE estava normal. A ultra-sonografia ocular diagnóstica (com sonda de $15 \mathrm{mHz}$, Mentor Instruments, Bromall, MA, USA) mostrou duas massas elevadas na coróide, localizadas nasal superior e inferior à papila, medindo cerca de 3,65 mm de espessura (lesão superior) e 4,25mm de espessura (lesão inferior) com descolamento não-regmatogênico da retina adjacente (Fig. 2B).

As hipóteses diagnósticas consideradas foram tumor ocular de provável origem metastática versus endoftalmite endógena.

Foi proposta a biópsia aspirativa com agulha fina (BAAF) via transvítrea da lesão ocular e biópsia da lesão pulmonar via broncoscopia uma vez que havia dúvida do clínico quanto à origem metastática da lesão. Devido à citologia obtida na biópsia pulmonar não ter um tipo celular característico (Fig. 3), optou-se por realizar a biópsia aspirativa com agulha fina (BAAF) em OD, 3 dias depois.

A BAAF ocorreu sem intercorrências exceto por uma hemorragia vítrea leve $(1+)$ que reabsorveu em 4 dias. Vinte e quatro horas depois o olho apresentava-se mais calmo e menos doloroso.

Devido ao resultado citológico coincidente da BAAF ocular e a citologia pulmonar (Fig. 3), optou-se pela quimioterapia sistêmica. Quarenta dias após BAAF foi realizada nova avaliação oftalmológica do paciente que já havia realizado 2 sessões de quimioterapia.

A acuidade visual medida foi: $O D=20 / 400$ com janela nasal e $\mathrm{OE}=20 / 20$. A PO medida foi $\mathrm{OD}=9 \mathrm{mmHg}$ e $\mathrm{OE}=10 \mathrm{mmHg}$ (15:35h). A biomicroscopia do segmento anterior revelou OD calmo, com hiperemia 1+, sem secreção e reflexo pupilar amarelado. OE apresentou-se dentro dos limites da normalidade. A oftalmoscopia binocular indireta do olho direito revelou regressão da massa superior e retina colada nesta região, um dos pontos biopsiados (superior) foi visualizado, e a retina adjacente estava aplicada (Fig. 5-esquerda). Entretanto ainda 

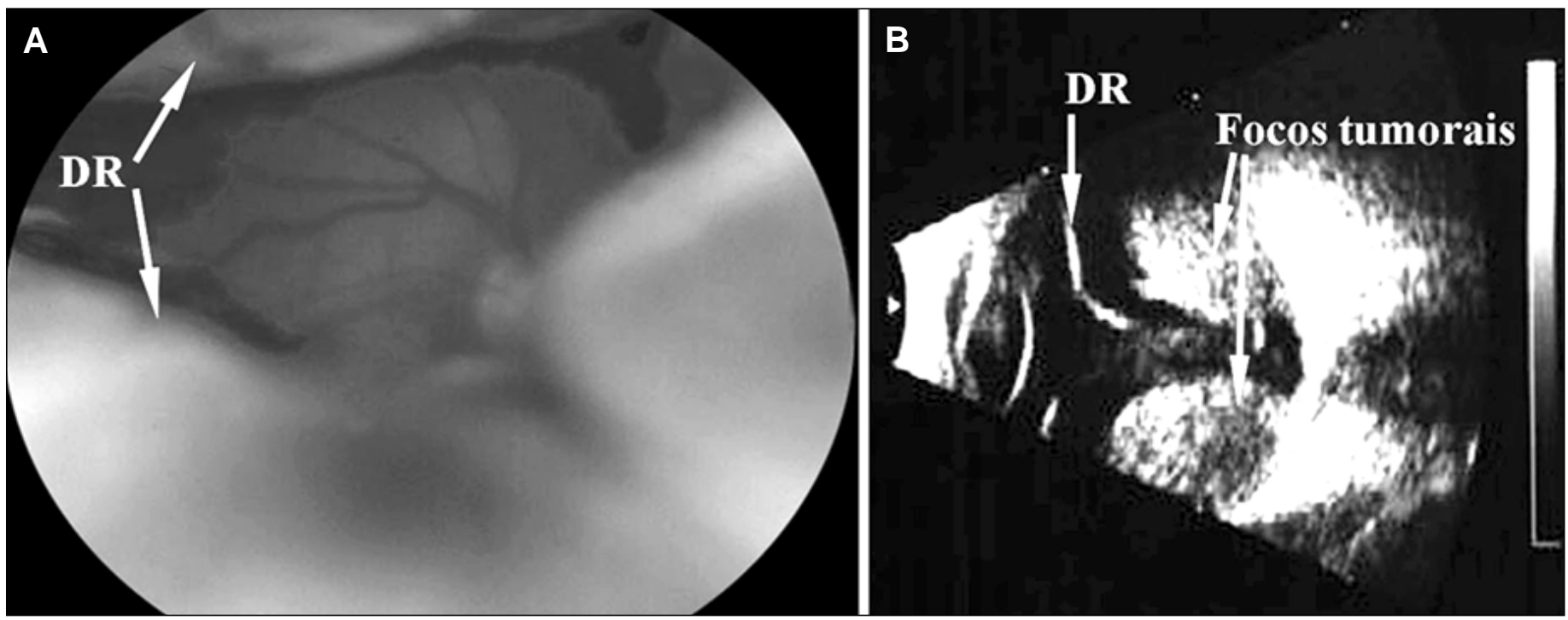

Figura 2 - Retinografia e ultra-sonografia de OD na consulta inicial. Note o exuberante descolamento de retina (DR) não-regmatogênico e com aspecto atípico (esquerda) e a presença de vários focos tumorais em coróide, superior e inferior ao disco óptico, sob a retina descolada (DR) (ultra-som à direita)

havia massa inferior com descolamento de retina nesta área. OE apresentou-se normal. A ultra-sonografia diagnóstica revelou massa intra-ocular inferior com algumas lacunas hiperecogênicas sugerindo necrose intratumoral. A espessura média desta lesão pelo ultra-som foi $3,25 \mathrm{~mm}$ e descolamento da retina persistente sobre a mesma (Fig. 5-direita). Na região superior observou-se apenas um espessamento da coróide sugerindo uma regressão tumoral satisfatória.

Dois meses depois, durante o tratamento com quimioterápicos, o paciente foi re-internado com dor torácica intensa e epistaxe, na investigação laboratorial foi detectada anemia severa. O quadro ocular permaneceu inalterado. O paciente evoluiu para óbito aproximadamente 4 meses após o diagnóstico do câncer metastático em coróide.

\section{MÉTODOS}

A técnica usada para a BAAF já foi previamente descrita ${ }^{(1-2)}$. Este procedimento tem sido realizado em ambiente cirúrgico sob cuidados de assepsia semelhantes àqueles usados em cirurgias intra-oculares. Foi usada uma agulha de gauge 23 e

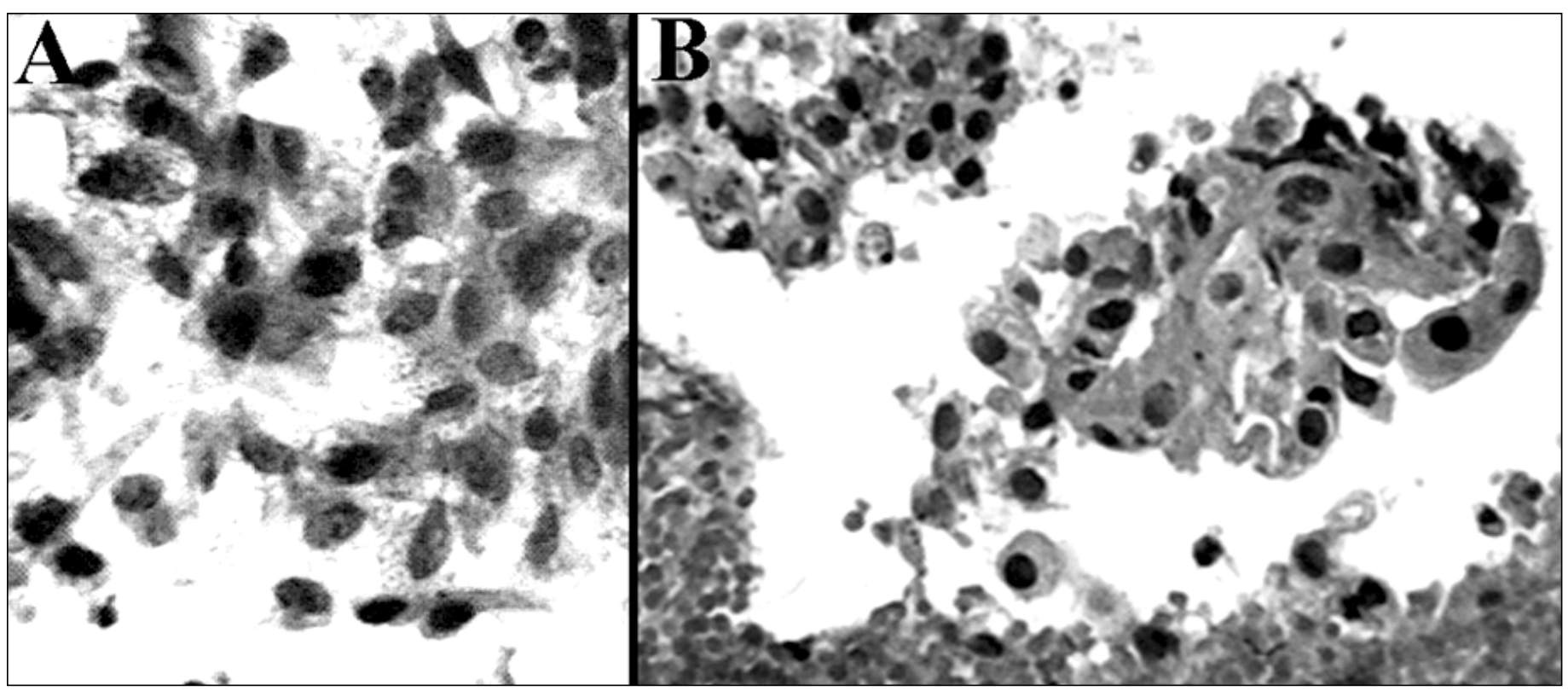

Figura 3 - Material obtido durante a BAAF da lesão intra-ocular (A) e na biópsia endo-brônquica (B). Coloração usada em ambas foi hematoxilinaeosina. Aumento 200X 
comprimento de $30 \mathrm{~mm}$, conectada a um segmento de equipo de soro com cerca de $40 \mathrm{~cm}$ que, por sua vez, estava conectado a uma seringa descartável de $10 \mathrm{ml}$ com a qual se realiza a aspiração. O procedimento foi monitorado via oftalmoscopia binocular indireta. A rota escolhida foi transvítrea através de uma esclerotomia a $4 \mathrm{~mm}$ do limbo nasal superior. Após bloqueio anestésico retrobulbar e sedação leve, a BAAF foi realizada em 2 pontos diferentes pela perfuração da retina nas áreas com menor quantidade de descolamento. A aspiração manual do conteúdo tumoral foi realizada pelo auxiliar. Após a aspiração, a agulha foi retirada num movimento contínuo seguindo trajeto semelhante ao que a mesma foi introduzida no olho seguido de suave compressão digital por cerca de 60 segundos para aumentar a pressão intra-ocular e diminuir o sangramento vítreo.

A seguir a agulha foi desconectada do tubo de silicone e conectada a uma seringa descartável de $5 \mathrm{ml}$ usada para aspirar 1-2 ml de solução salina balanceada com objetivo de lavar o conteúdo celular contido no lúmen da agulha.

O mesmo procedimento foi então repetido para colher material de outra região do tumor. O material obtido nas 2 amostras foi encaminhado imediatamente para processamento, cito-centrifugado, fixação e coloração tipo Papanicolau e hematoxilina-eosina no laboratório de patologia.

Além destes, foram usados anticorpos para citoqueratina (AE1/AE3) para confirmar a presença de células tumorais tipo carcinoma (neoplasia maligna de origem epitelial) metastático e o HMB-45 para afastar a possibilidade de ser um melanoma maligno primário da coróide.

\section{RESULTADOS}

Descrição Cito-patológica (Fig. 3)

\section{Broncoscopia}

Biópsia endobrônquica mostra neoplasia maligna nãopequenas células (escasso tecido neoplásico com necrose $\mathrm{e}$ distorções artefaturais), provável adenocarcinoma.

Linfonodos subcarinais, paratraqueais, inferiores direitos apresentam linfadenite crônica com antracose.

\section{Laudo da BAAF do olho direito}

Em um dos dois esfregaços identifica-se um grupamento de células pleomórficas com núcleo hipercromático e características sugestivas de malignidade. Diagnóstico: neoplasia maligna indiferenciada, compatível com carcinoma metastático.

\section{Imuno-citoquímica (Fig. 4)}

Foram realizados testes HMB-45 e pan-queratina (AE1/ AE3) sendo que ambos aspirados ocular e pulmonar apresentaram-se AE1/AE3 positivos e HMB-45 negativos.

Considerando estes achados, o diagnóstico final foi carcinoma broncogênico não-pequenas células e metástase ocular direita.

\section{DISCUSSÃO}

O carcinoma metastático no olho é a neoplasia intra-ocular

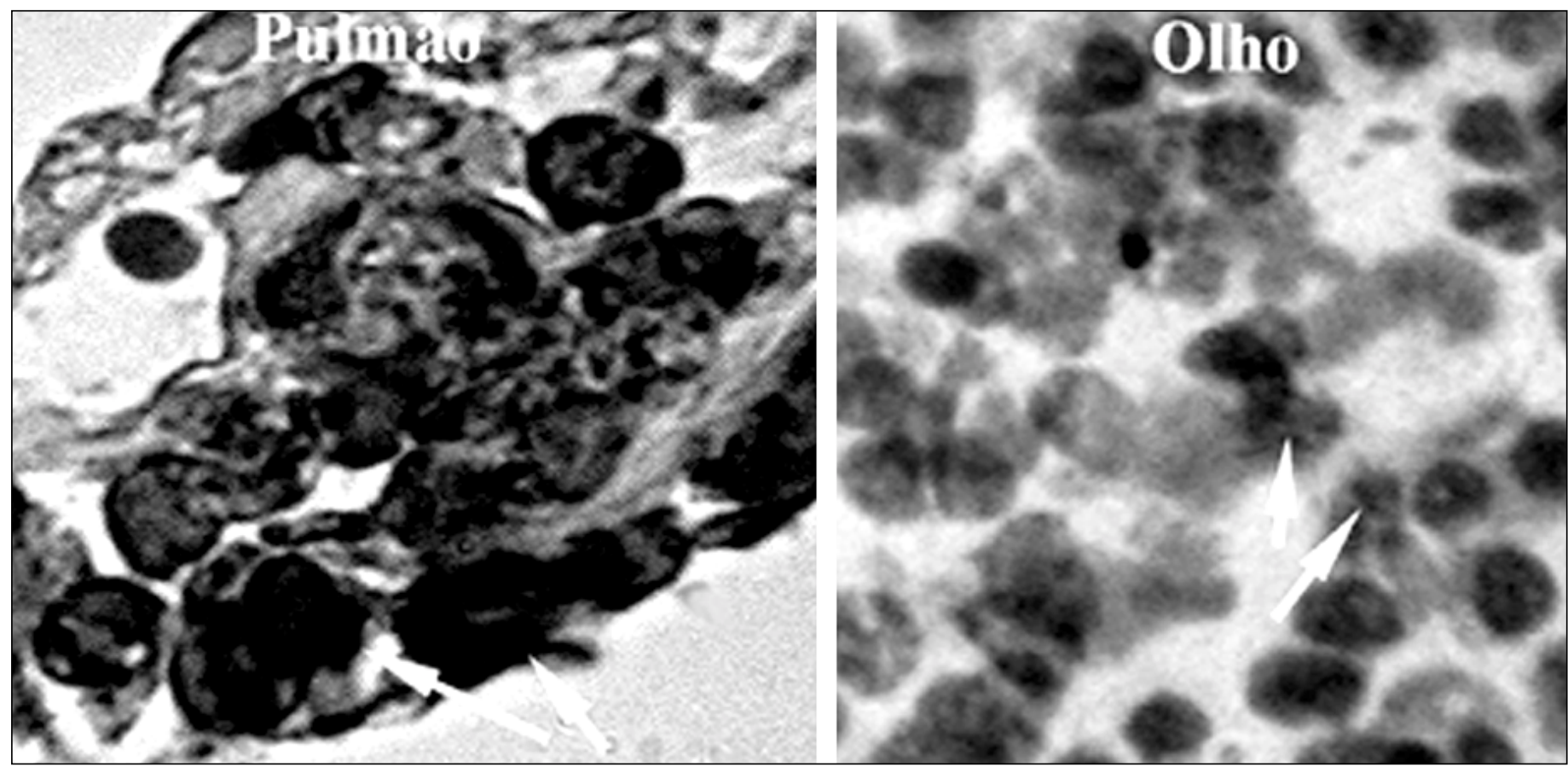

Figura 4 - Aspecto do material com técnica de imuno-citoquímica, pan-queratina + (AE1/AE3). Devido à fotografia ser preto-branco as áreas mais escuras (apontadas por flechas) são as que apresentaram uma coloração acastanhada (AE1/AE3+) quando comparada ao restante da lâmina. Aumento $400 \mathrm{X}$ 
mais prevalente entre os adultos. O sítio primário mais comum é mama nas mulheres e o pulmão entre os homens ${ }^{(9)}$, como ocorreu no caso descrito. A maioria dos tumores metastáticos intra-oculares envolve a coróide na proporção 8:1 em relação às metástases orbitárias ${ }^{(10)}$. Aproximadamente $80 \%$ das pessoas afetadas apresentam-se com tumor único em um olho, enquanto que os $20 \%$ restantes têm tumores múltiplos, bilaterais ou os dois ${ }^{(1)}$. As metástases oculares na coróide evoluem em $75 \%$ dos casos com descolamento seroso da retina sensorial muito mais extenso que a massa tumoral. O comprometimento macular depende da localização tumoral ${ }^{(9)}$. Alguns casos de metástases oculares de carcinoma broncogênico foram descritas $^{(10-13)}$, cada uma com suas particularidades de apresentação e evolução que salienta a multiplicidade de apresentações deste tumor. Nesse caso, o paciente apresentou múltiplos focos tumorais e descolamento de retina não-regmatogênico (exsudativo) exuberante comprometendo parcialmente a mácula. Na oftalmoscopia binocular indireta, as metástases de coróide costumam se apresentar como uma infiltração difusa com características semelhantes àquelas da neoplasia primária. Apesar disto, o grau de diferenciação celular torna a identificação do sítio primário da neoplasia difícil ou por vezes, impossível $^{(1)}$. A ultra-sonografia do carcinoma metastático de coróide $^{(9)}$ caracteriza-se por ecos de alta amplitude e intensa refletividade da lesão que geralmente não é muito espessa quando comparada ao exuberante descolamento de retina associado. Apesar da presença de dois focos tumorais em um olho ser um achado altamente sugestível de metástase ocular; a dificuldade de avaliar características tumorais pelo descolamento exsudativo da retina, o quadro clínico sistêmico não compatível com doença metastática e a importância deste diagnóstico na terapia sistêmica do paciente levaram à indicação da BAAF para elucidação diagnóstica.

Vários fatores devem ser considerados no planejamento da biópsia aspirativa com agulha fina como: tipo de tumor, tamanho, localização, extensão do descolamento de retina associado e a transparência dos meios ${ }^{(1)}$. Os tumores metastáticos do segmento posterior (coróide) podem ser biopsiados via transescleral ou transvítrea ${ }^{(1-2)}$, sendo a segunda mais utilizada. A observação da lesão durante o procedimento pode ser direta (microscópica) ou indireta (oftalmoscópio binocular indireto $)^{(1)}$. A escolha do local de entrada se deu com o objetivo de propiciar a melhor observação do trajeto da agulha durante a BAAF, não somente pela localização do tumor, mas também pelas condições sistêmicas do paciente que foi submetido ao procedimento sentado à frente da cirurgiã. Não é obrigatório que o local de entrada da agulha seja oposto à lesão uma vez que já foram descritos procedimentos trans-esclerais ${ }^{(1)}$, entretanto um ângulo mínimo de 30 a 45 graus entre a lesão e o sítio de entrada da agulha tem dado bons resultados na experiência dos autores. Portanto a opção foi pela via transvítrea com observação indireta. $\mathrm{O}$ número de amostras colhidas varia de acordo com o cirurgião e costuma ser no mínimo duas ${ }^{(3,5)}$. O preparo e coloração dos aspirados segue a mesma rotina dos preparos citológicos usados em amostras de outros órgãos. O uso da imuno-citoquímica no diagnóstico e diferenciação de células neoplásicas tem sido amplamente explorado e deve ser considerado em casos semelhantes a esse.

Alguns aspectos devem ser citados quando se considera biopsiar uma lesão intra-ocular suspeita de malignidade: 1- as complicações potenciais deste procedimento como hemorra-
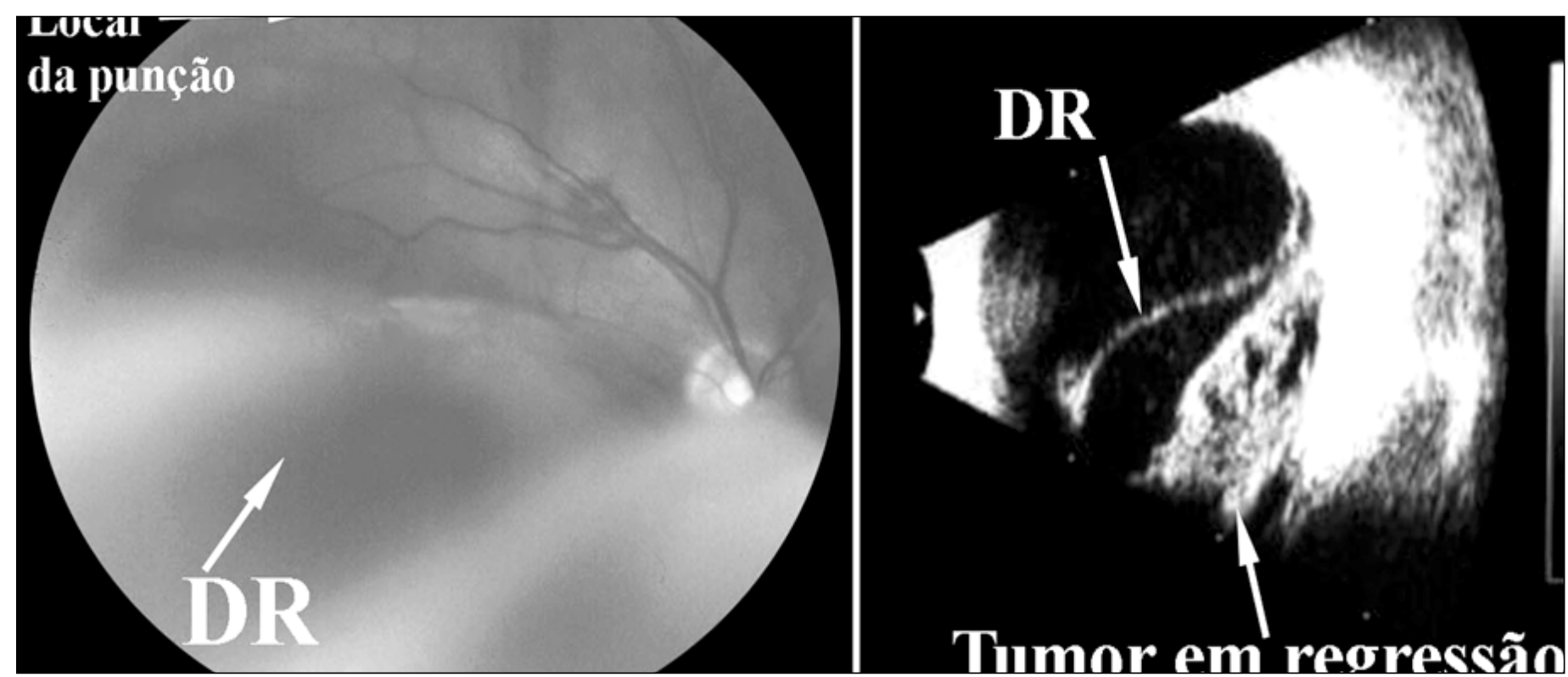

Figura 5 - Retinografia e ultra-sonografia 2 meses após BAAF e diagnóstico de tumor metastático de coróide. Note a completa regressão do foco tumoral superior (no local onde foi feita a primeira punção) na imagem à esquerda. Na ultra-sonografia observa-se que o tumor residual inferior apresenta lacunas em seu interior sugerindo a presença de áreas de necrose (direita) e o descolamento de retina permanece desproporcional comparado ao tamanho da lesão 
gia vítrea, descolamento de retina, endoftalmite e catarata traumática, 2- a possível (mas ainda não comprovada) disseminação de células tumorais, 3 - a experiência do oftalmologista que realizará o procedimento e o patologista que interpretará o material aspirado e 4- a repercussão da quantidade insuficiente de material para diagnóstico citológico. Cada um destes aspectos merece uma infindável discussão que não cabe neste artigo.

A biópsia aspirativa com agulha fina (BAAF) foi eficiente em diagnosticar e correlacionar a citologia ocular com o tumor primário por métodos citoquímicos neste caso. A BAAF ainda deve ser usada em casos selecionados e pesquisas futuras serão necessárias para que este procedimento diagnóstico seja considerado padrão em oftalmologia.

\section{ABSTRACT}

Purpose: To report a case of a patient with lung carcinoma in which the first detected metastasis was to the choroid, how it was diagnosed and confirmed. Methods: A 35 year-old white male, while being treated for a solitary pulmonary condensation, reported sudden loss of vision, pain, discharge and red eye (right eye) for 10 days. During the ophthalmic examination a nonregmatogenous retinal detachment as well as multiple choroidal tumors were confirmed by diagnostic ocular ultrasound. Fine-needle aspiration biopsy (FNAB) was suggested to diagnose a possible metastatic disease. Fine-needle aspiration biopsy was performed under peribulbar anesthesia with sedation. A transvitreous route was chosen through a sclerotomy $4 \mathrm{~mm}$ from the limbus. The procedure was monitored via binocular indirect ophthalmoscopy. Two sample aspirates were obtained from different tumour foci. After fine-needle aspiration biopsy, the aspirates were sent for processing, fixation and stained with Papanicolaou and HE. Results: Cytology confirmed the diagnosis of multiple metastatic tumors. Immunocytochemistry of ocular and lung aspirates revealed a common cell origin by a pankeratin (AE1/AE3) positive test. Regardless of systemic treatment with chemotherapy and improvement of the ocular status, the patient died 4 months after cytological diagnosis of metastatic carcinoma of the choroid. Conclusions: Fine-needle aspiration biopsy was efficient to diagnose and correlate ocular cytology with the primary tumor by imunohistochemical methods in this case. Fine-needle aspiration biopsy should still be used only in selected cases and further research will be necessary for it to become a standard diagnostic procedure in ophthalmology.

Keywords: Lung neoplasms/complications; Choroid neoplasms/secondary; Choroid neoplasms/pathology; Immunohistochemistry; Ultrasonography; Needle biopsy; Eye/cytology; Carcinoma/pathology; Eye neoplasms/pathology; Hematoxylin/diagnostic use; Eosine yellowish-(YS)/diagnostic use; Case report

\section{REFERÊNCIAS}

1. Augsburger JJ, Shields JA. Fine needle aspiration biopsy of solid intraocular tumours: indications, instrumentation and techniques. Ophthalmic Surg 1984;15:34-40.

2. Augsburger JJ, Shields JA, Folberg R, Lang W, O'Hara BJ, Claricci JD. Fine needle aspiration biopsy in the diagnosis of intraocular cancer: Cytologic-histologic correlations. Ophthalmology 1985;92:39-49.

3. Augsburger JJ. Fine needle aspiration biopsy of suspected metastatic cancer to the posterior uvea. Trans Am Ophthalmol Soc 1988;86:499-560.

4. Jakobiek FA, Coleman DJ, Chattock A, Smith M. Ultrasonically guided needle biopsy and cytologic diagnosis of solid intraocular tumours. Ophthalmology 1979;86:1662-81.

5. Augsburger JJ, Shields JA, Lang W. Fine needle aspiration biopsy of intraocular tumours. Invest Ophthalmol 1986;27:260.

6. Ritland JS, Eide N, Hoie J. Fine-needle aspiration biopsy diagnosis of a uveal metastasis from a follicular thyroid carcinoma. Acta Ophthalmol Scand 1999;77:594-6.

7. Corrêa ZMS, Marcon IM. O uso da biópsia aspirativa com agulha fina no diagnóstico de lesão iriana suspeita. Arq Bras Oftalmol 2001;64:347-50.

8. Eide N, Syrdalen P, Walaas L, Hagmar B. Fine-needle aspiration biopsy in selecting treatment for inconclusive intra-ocular disease. Acta Ophthalmol Scand 1999;77:448-52.

9. Castro E, Vianna RNG, Ávila M. Metástases intra-oculares. In: Abujamra S, Ávila M, Barsante C. editores. Retina e vítreo: clínica e cirurgia. São Paulo: Roca; 2000. p.398-402.

10. Shields JA, Shields CL, Eagle Jr RC, Gunduz K, Lin B. Diffuse ocular metastases as an initial sign of metastatic lung cancer. Ophthalmic Surg Lasers 1998;29:598-601.

11. Police G, Perri P, Pastena B, Ravalli L, Grande L, Rossi A. Rapidly growing choroidal metastasis: case report. Ophthalmologica 1998;212(suppl 1):37-9.

12. Buys R, Abramson DH, Kitchin FD, Gottlieb F, Epstein M. Simultaneous ocular and orbital involvement from metastatic bronchogenic carcinoma. Ann Ophthalmol 1982;14:1165-7, 1170-1.

13. Latkany P, Ciulla TA, Younger C, Gao H. Nonsmall cell adenocarcinoma metastatic to the vitreous without fundus lesions. Ophthalmic Surg Lasers 2000;31:155-6.

\section{Ao enviar um artigo para publicação, leia ATENTAMENTE as instruções para autores, constante no final de cada fascículo.}

